\title{
Modelling of the Thermodynamic Properties of the Plasma Mixture
}

\section{A. S. Polyukhin}

Numerical modelling of the thermodynamic properties of plasma mixture is performed using the Thomas-Fermi model with two different approaches. For this purpose, a numerical algorithm, as well as program realization, is developed to solve the Thomas-Fermi equations with quantum-exchange corrections. For the first time a comparison between different methods for taking account of the heterogeneous composition of plasma is made and an algorithm for estimating the corrections for mixtures is developed.

Keywords: computational model, magnetized plasma, numerical method, thermodynamic property

\section{Introduction}

The main purpose of this work is to develop an algorithm for modelling thermodynamic properties of plasma mixtures in a magnetic field using the Thomas-Fermi model [1] with quantum and exchange corrections and to compare different approaches to the given problem. All calculations are made by employing two different approaches: by solving the system of equations of the Thomas-Fermi model with the condition of thermodynamic equilibrium and by using average ion approximation.

Received January 25, 2021

Accepted March 04, 2021

\footnotetext{
Alexander S. Polyukhin

polyukhinas@gmail.com

Joint Institute for High Temperatures of the Russian Academy of Sciences

ul. Izhorskaya 2, Moscow, 125412 Russia

Moscow Institute of Physics and Technology

Institutskiy per. 9, Dolgoprudny, Moscow Region, 141701 Russia
} 
Correct and accurate modeling of plasma thermodynamic parameters is a complex and timeconsuming task. The analysis of the Thomas - Fermi model with quantum-exchange corrections, comparison with other models and its extension to the region of multicomponent systems was presented earlier, but not in such a wide range of parameters.

In this paper, we study the thermodynamic parameters of one-component and multicomponent plasma in an external magnetic field based on the Thomas-Fermi model with quantumexchange corrections. All data are calculated in the region of temperatures of $10 \mathrm{eV}<T<10^{5}$ since it is a preferable area for the problems of fusion energy [2].

\section{Numerical methods}

The main idea of the first approach is solving the system of Thomas - Fermi equations [3] for each component of mixture ( $N$ substances) with the additional condition that the sum of components' volumes is equal to the volume of the whole system:

$$
\frac{1}{\rho} \sum_{i} m_{i}=\sum_{i} \frac{m_{i}}{\rho_{i}}
$$

where $m_{i}$ is a mass fraction of the component, $\rho_{i}$ is partial density of the component, and $\rho$ is average density. Using the condition of thermodynamic equilibrium that sets equality of the chemical potential of each component of the system, one can obtain a system of equations which can be used to determine potential $\phi$ :

$$
\begin{gathered}
\frac{d^{2} \phi_{i}}{d x^{2}}=a_{i} x I_{1 / 2}\left(\frac{\phi_{i}}{x}\right) \quad(i=1,2, \ldots, N), \\
\phi_{i}^{\prime}(1)=\left.\frac{d \phi_{i}}{d x}\right|_{x=1}=\frac{\mu_{i}}{\theta}=-\eta_{i}, \\
\phi_{i}(0)=\frac{Z_{i}}{\theta r_{0 i}}, \\
\eta_{i}=\eta_{j}=-\frac{\mu}{\theta} \quad(\forall i, j), \\
x=\frac{r}{r_{0 i}}, \quad \frac{\phi_{i}(x)}{x}=\frac{V_{i}(r)+\mu_{i}}{\theta}, \\
r_{0 i}=1,388\left(\frac{A_{i}}{\rho_{i}}\right)^{1 / 3}, \quad a_{i}=\frac{4 \sqrt{2 \theta}}{\pi} r_{0 i}^{2}, \\
I_{k}(x)=\int_{0}^{\infty} \frac{y^{k} d y}{1+\exp (y-x)},
\end{gathered}
$$

where $V_{i}(r)$ is electrostatic potential, $\phi$ is reduced electrostatic potential, $\mu_{i}$ is chemical potential, $\eta_{i}$ is reduced chemical potential, $r_{0 i}$ is the boundary radius of an atomic cell, $Z_{i}$ is the charge of a nucleus, $A_{i}$ is atomic weight, and $I_{k}(x)$ is the Fermi-Dirac function. Here and in what follows, all values with index $i$ correspond to the simple substance of the mixture. Radii of atomic cells should be chosen using (2.1) and the condition of thermodynamic equilibrium. Using linearization and additional transformations, the system (2.2) can be solved numerically using iteratively the sweep method [4]. 
After estimation of potential $\phi$ all basic thermodynamic functions of selected species can be calculated according to the Thomas-Fermi model by the following relations [5]:

$$
\begin{gathered}
S_{e}=\frac{4 \sqrt{2} \theta^{3 / 2} r_{0}^{3}}{\pi}\left[\frac{4}{3} I_{3 / 2}(-\eta)-\frac{7}{3} \int_{0}^{1} x^{2} I_{3 / 2}\left(\frac{\phi(x)}{x}\right) d x\right]-Z \phi^{\prime}(0), \\
P_{e}=\frac{(2 \theta)^{5 / 2}}{6 \pi^{2}} I_{3 / 2}(-\eta), \\
E_{e}=E_{p e}+E_{k e} \\
E_{k e}=\frac{3 \sqrt{2}}{(\pi)^{2}} \nu \theta^{5 / 2} \int_{0}^{1} x^{2} I_{3 / 2}\left(\frac{\phi(x)}{x}\right) d x \\
E_{p e}=\frac{2 \sqrt{2}}{\pi^{2}} \nu \theta^{5 / 2}\left[I_{3 / 2}(-\eta)-3 \int_{0}^{1} x^{2} I_{3 / 2}\left(\frac{\phi(x)}{x}\right) d x\right]
\end{gathered}
$$

where $\eta=-\phi(1), \nu=\frac{4}{3} \pi r_{0}^{3}$, and $S_{e}, P_{e}, E_{e}$ are thermodynamic functions of the electron system. The pressure, internal energy, and entropy of the nuclear component are determined by the classical formulas of an ideal gas. In order to get thermodynamic properties of the system, the sum of the components of the mixture should be carried out:

$$
\begin{array}{r}
E=\frac{\sum_{i} E_{i} h_{i} A_{i}}{\sum_{i} h_{i} A_{i}}, \\
S=\frac{\sum_{i} S_{i} h_{i} A_{i}}{\sum_{i} h_{i} A_{i}},
\end{array}
$$

where $S$ is the entropy of the system, $E$ is the internal energy of the system, $h_{i}$ is the mole fraction of the $i$ component, $S_{i}$ is the entropy of the $i$ component, and $E_{i}$ is the internal energy of the $i$ component. The pressure of the system can be found using the relation of the classical Thomas - Fermi model because the pressure of all components is the same due to the condition of thermodynamic equilibrium. Thermodynamic consistency is achieved because the pressure depends on chemical potential, which is the same for all species due to condition (2.2).

Introduction of quantum-exchange corrections in the Thomas-Fermi model for mixtures allows expanding the limit of applicability in the region of lower temperatures. The method that provides corrections is based on the use of the quantum mechanical approach in the derivation of the Thomas - Fermi equation [6]. The theory provides the following equation for correction of the potential:

$$
\begin{gathered}
\chi_{i}^{\prime \prime}-\frac{4 \sqrt{2 \vartheta}}{\pi} r_{0 i}^{2} I_{1 / 2}\left(\frac{\phi_{i}(x)}{x}\right) \chi_{i}=\frac{4 \sqrt{2 \vartheta}}{\pi} r_{0 i}^{2} x Y^{\prime}\left(\frac{\phi_{i}(x)}{x}\right), \\
\chi_{i}(0)=0, \quad \chi_{i}^{\prime}(1)=1,
\end{gathered}
$$

where $\chi_{i}$ is the reduced correction of the Thomas - Fermi potential and $Y=I_{1 / 2}(x) I_{1 / 2}^{\prime}(x)+$ $+6 \int_{-\infty}^{x}\left[I_{1 / 2}^{\prime}(t)\right]^{2} d t$. In this approach, the correction for pressure, internal energy and entropy 
can be written as

$$
\begin{aligned}
& \Delta P_{i}=\frac{\vartheta^{2}}{3 \pi^{3}}\left[\chi_{i}(1) I_{1 / 2}\left(\phi_{i}(1)\right)+Y\left(\phi_{i}(1)\right)\right], \\
& \Delta E_{i}=\frac{\vartheta^{2}}{3 \pi^{2}} r_{0 i}^{2}\left[\int_{0}^{1} x \chi_{i}(x) I_{1 / 2}\left(\frac{\phi_{i}(x)}{x}\right) d x+2 \int_{0}^{1} x^{2} Y\left(\frac{\phi_{i}(x)}{x}\right) d x\right]+\frac{Z \sqrt{2 \theta}}{6 \pi} \chi_{i}^{\prime}(0)+0,2690017 Z^{5 / 3}, \\
& \Delta S_{i}=\frac{2 \vartheta}{3 \pi^{2}} r_{0 i}^{2}\left[\int_{0}^{1} x \chi_{i}(x) I_{1 / 2}\left(\frac{\phi_{i}(x)}{x}\right) d x+4 \int_{0}^{1} x^{2} Y\left(\frac{\phi_{i}(x)}{x}\right) d x\right]+\frac{Z \sqrt{2}}{6 \pi \sqrt{\vartheta}} \chi_{i}^{\prime}(0),
\end{aligned}
$$

where $\Delta P_{i}, \Delta E_{i}, \Delta S_{i}$ are quantum-exchange corrections of thermodynamic functions. Since the total contribution of corrections for thermodynamic functions requires sums such as in relations (2.3) and (2.4), it will inevitably violate the condition of thermodynamic equilibrium. For equilibrium, the system should have equal values of pressure and chemical potential for each species, which cannot be achieved because correction for these thermodynamic properties is individual for every component of the mixture. It should be mentioned that the values of these corrections depend greatly on temperature and density, so in the region of high temperatures and densities the corrections become negligible and the condition of thermodynamic equilibrium will be performed automatically.

Another approach of mixtures' modelling is the average ion approximation [7]. This method was first applied to a similar problem of determining the thermodynamic properties using the Saha ionization model. The main idea is to replace the original system of various ions with a new system consisting of ions averaged over all components:

$$
Z=\sum_{i} h_{i} Z_{i}, \quad A=\sum_{i} h_{i} A_{i} .
$$

To calculate thermodynamic properties and their corrections using the Thomas-Fermi model, an average ion approximation can be used as well by replacing the system of equations (2.2) with only one equation of an averaged cell. In this approximation, the whole mixture can be described by the following system:

$$
\begin{gathered}
\frac{d^{2} \phi}{d x^{2}}=a x I_{1 / 2}\left(\frac{\phi}{x}\right), \\
\phi^{\prime}(1)=\left.\frac{d \phi}{d x}\right|_{x=1}=\frac{\mu}{\theta}=-\eta, \\
\phi(0)=\frac{Z}{\theta r_{0}}, \\
\chi^{\prime \prime}-\frac{4 \sqrt{2 \vartheta}}{\pi} r_{0}^{2} I_{1 / 2}\left(\frac{\phi(x)}{x}\right) \chi=\frac{4 \sqrt{2 \vartheta}}{\pi} r_{0}^{2} x Y^{\prime}\left(\frac{\phi(x)}{x}\right), \\
\chi(0)=0, \quad \chi^{\prime}(1)=1,
\end{gathered}
$$

where $\phi$ is the reduced Thomas - Fermi potential for the averaged ion and $\chi$ stands for reduced corrections of the Thomas-Fermi potential for the averaged ion.

Thermodynamic functions can be calculated using relations for the classical model with the Thomas - Fermi potential of the averaged ion. This approach satisfies the condition of thermodynamic equilibrium since each species will have equal values of average chemical potential, thus all thermodynamic properties for all species of the mixture will be equal. The accuracy of this approach should be established by comparing the results with the existing data. 


\section{Results and discussion}

To model the thermodynamic properties of plasma mixtures that could be used in problems of thermonuclear fusion, two mixtures were chosen: $60 \%$ gold $+40 \%$ silver and $90 \%$ beryllium + $10 \%$ tungsten. The first mixture of heavy cores can be implemented as a target component for problems of inertial and magnetic fusion [8-11]. The second one can be used in the creation of diverters and blankets of tokamaks. It should be mentioned that calculations performed in this work could be applied to a system with a strong magnetic field as it does not affect the present computations [12].

Figure 1 corresponds to the determination of pressure in the isochoric process $\left(\rho=1 \mathrm{~g} / \mathrm{cm}^{3}\right)$ as a function of temperature using different approaches. It is seen that all methods give similar results. However, considering quantum-exchange corrections reduce the pressure in the region of low temperature, since these corrections have a negative value. Differences between the method of taking account of the heterogeneity of plasma using the average ion approximation and the approach based on solving the system of equations with the condition of thermodynamic equilibrium are not significant. This fact is in direct agreement with the theory since a negative pressure is described by repulsive forces of exchange interactions. Such forces are completely ignored in the classical Thomas-Fermi model because their contribution is not significant at high temperatures.

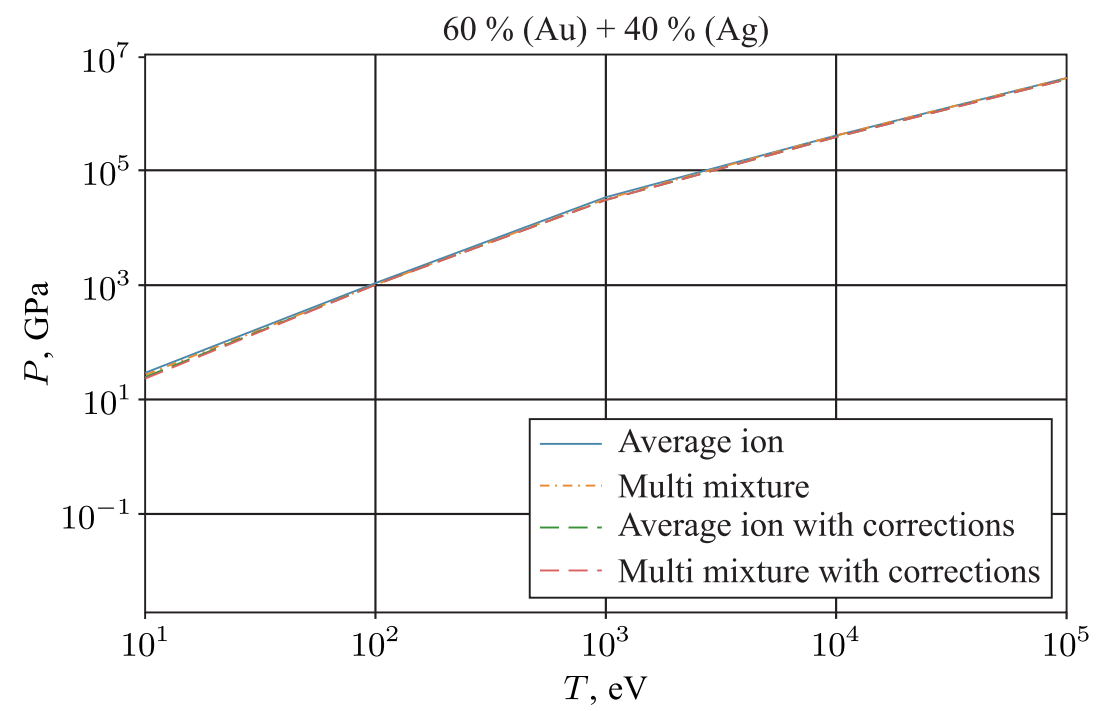

Fig. 1. Pressure of the mixture of gold and silver, based on the Thomas - Fermi model with and without quantum-exchange correction at a density of $1 \mathrm{~g} / \mathrm{cm}^{3}$.

Considering the solution method of the system of Thomas-Fermi equations with the additional condition of thermodynamic equilibrium, it can be inferred that, as mentioned above, taking account of quantum-exchange correction violates the condition of equilibrium. However, modelling shows that this approach insignificantly changes the result for heavy cores with a temperature higher than $10 \mathrm{eV}$.

The second mixture has a completely different picture of the distribution of thermodynamic functions. The main reason is variation in charge between the components of the mixture. Since the difference between the core charge of tungsten and that of beryllium is significant, the difference in averaging is much more serious. This fact leads to the results shown in Fig. 2. The 


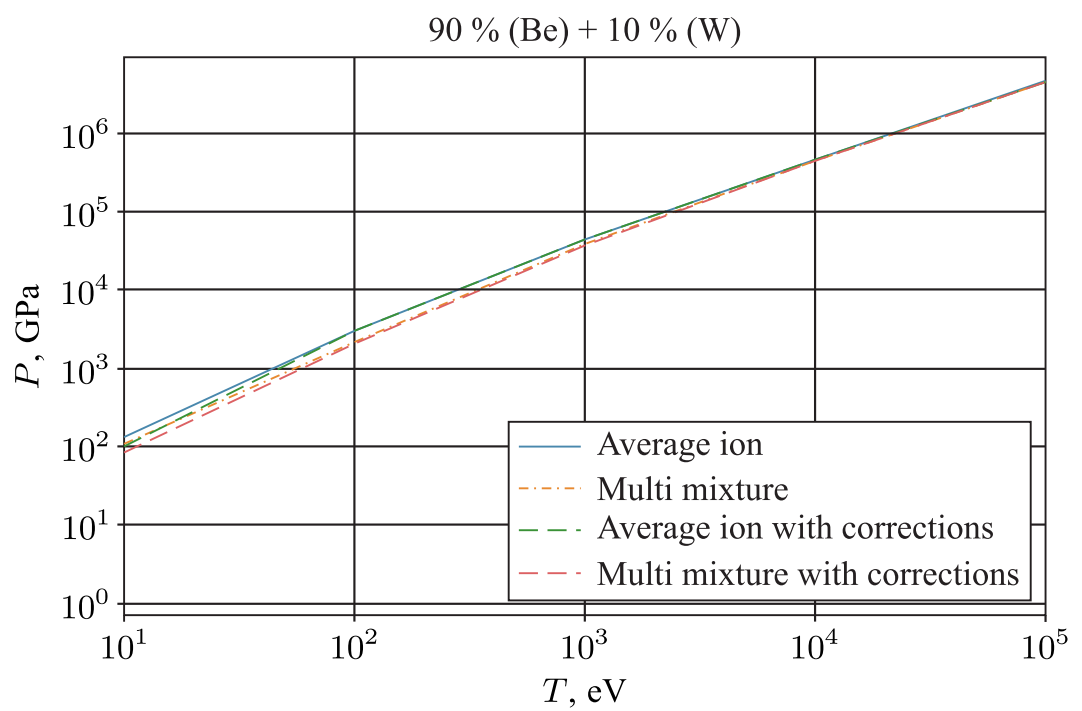

Fig. 2. Pressure of the mixture of beryllium and tungsten, based on the Thomas - Fermi model with and without quantum-exchange correction at a density of $1 \mathrm{~g} / \mathrm{cm}^{3}$.

method of solving equations of the Thomas - Fermi model with the additional condition of thermodynamic equilibrium usually gives lower values as compared to the average ion approximation. In the region of high temperatures, quantum-exchange corrections are negligible, all methods give the same values.

The modelling values of internal energy for the first mixture are almost equal regardless of the method of averaging species and taking into account the quantum-exchange corrections. The results presented in Fig. 3 can be explained by the fact that the contribution of the quantum exchange corrections for the internal energy at the chosen density begins to appear at much lower temperatures $(T<10 \mathrm{eV})$, as shown in Fig. 4. Since the plasma for the problems of

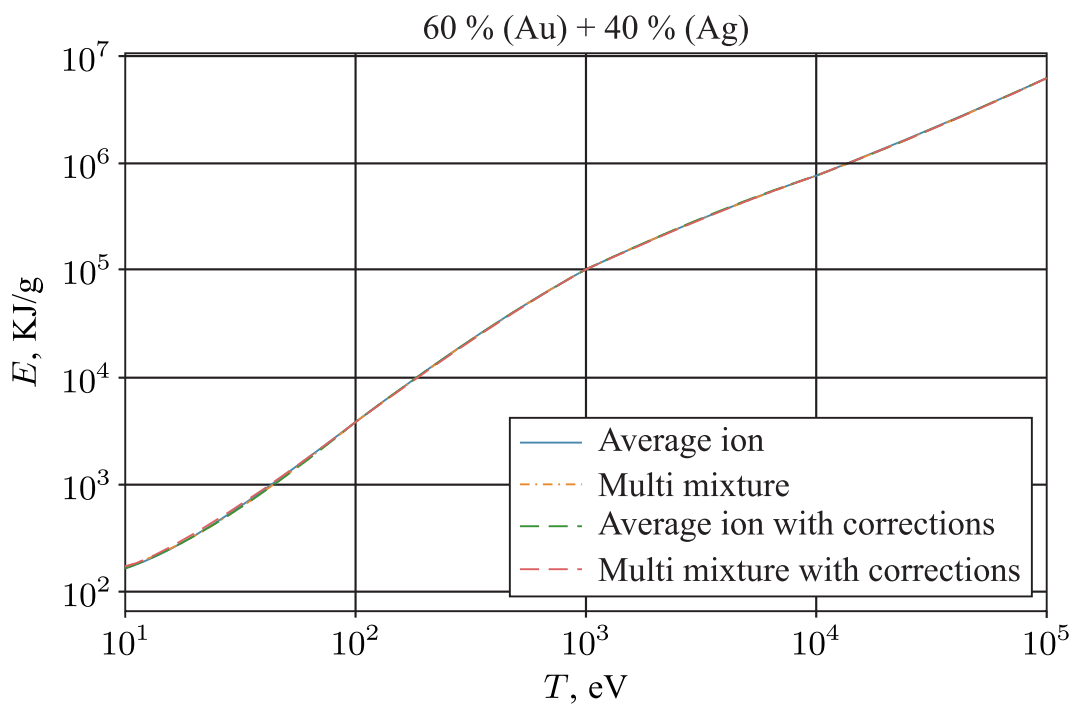

Fig. 3. Internal energy of the mixture of gold and silver, based on the Thomas - Fermi model with and without quantum-exchange correction at a density of $1 \mathrm{~g} / \mathrm{cm}^{3}$. 


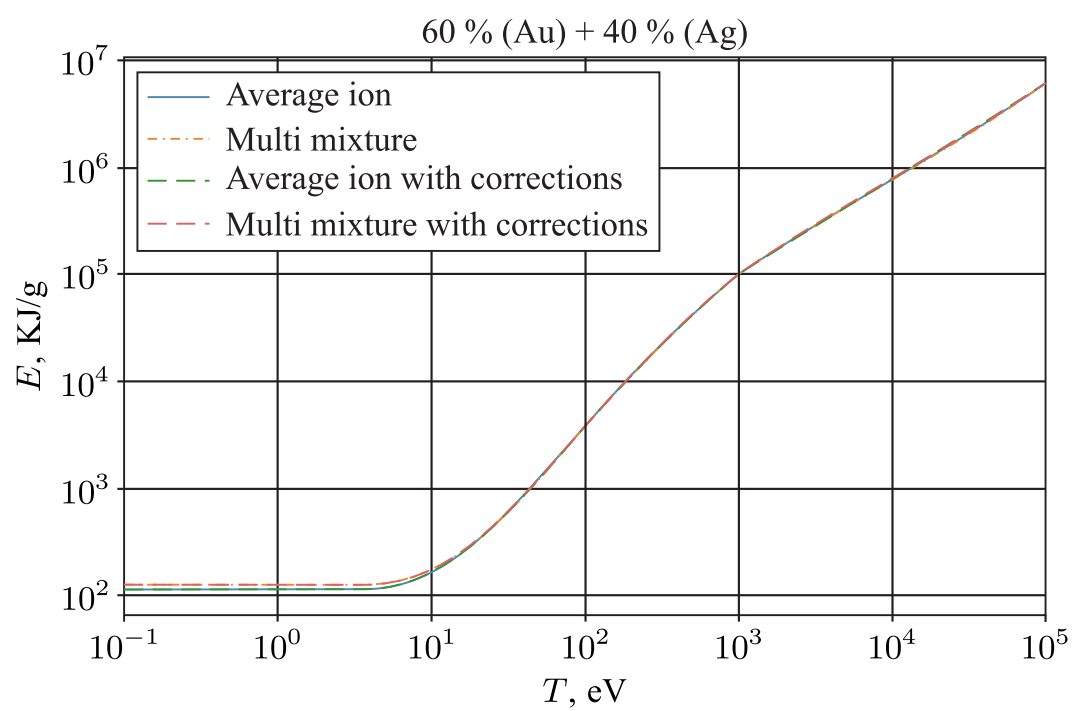

Fig. 4. Internal energy of the mixture of gold and silver, based on the Thomas - Fermi model with and without quantum-exchange correction at a density of $1 \mathrm{~g} / \mathrm{cm}^{3}$, in a wider temperature range.

thermonuclear fusion is not used at such low temperatures, the calculation gives similar values, regardless of whether or not the quantum-exchange corrections are taken into account.

Considering the values of internal energy in the second mixture, one can draw similar conclusions, as in the case of the calculation of pressure. The values obtained using the same method do not differ when considering the quantum-exchange corrections for the reasons described above. But the values obtained by different methods are different and this is seen in Fig. 5. As in the case of pressure, the difference between the charges of the beryllium and tungsten nuclei leads to a strong variation of internal energy values, achieved by two approaches of averaging.

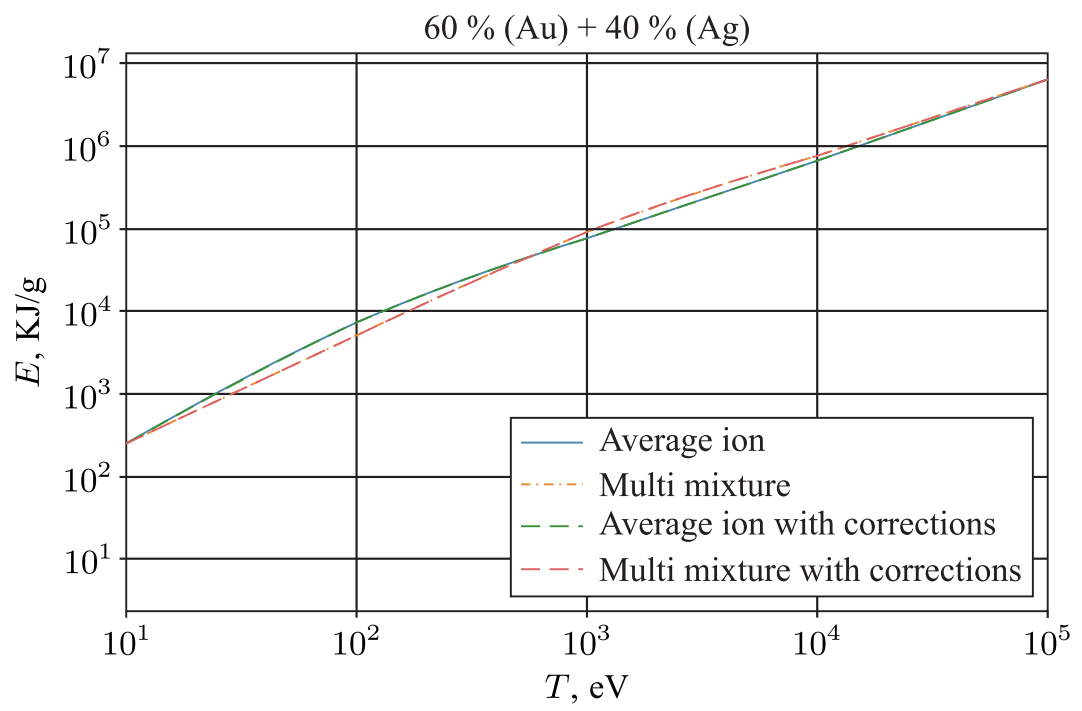

Fig. 5. The internal energy of the mixture of beryllium and tungsten, based on the Thomas - Fermi model with and without quantum-exchange correction at a density of $1 \mathrm{~g} / \mathrm{cm}^{3}$. 
All the arguments related to the internal energy can also be referred to the entropy of the system described in Fig. 6. In the region of relatively low temperatures, significant errors in the calculated values of entropy with corrections may appear due to the use of a simple approximation for the Fermi-Dirac functions, while there are more complex analogues [13, 14]. It is shown in $[15,16]$ that the calculation of internal energy and entropy according to the Thomas - Fermi model is less accurate than that of pressure, so in the temperature range $(T<100 \mathrm{eV})$, quantum exchange corrections can give nonphysical results. Such an approximation of the Fermi-Dirac functions was used since the plasma temperatures suitable for problems of thermonuclear fusion are much higher than the value of $100 \mathrm{eV}$. Moreover, areas where quantum-exchange corrections reach the value of a thermodynamic function should not give a physical result, since the correction is derived under the assumption of being small relative to the thermodynamic function it corrects. At high temperatures $(T>10 \mathrm{keV})$, all the methods give similar results for evaluation of the entropy.

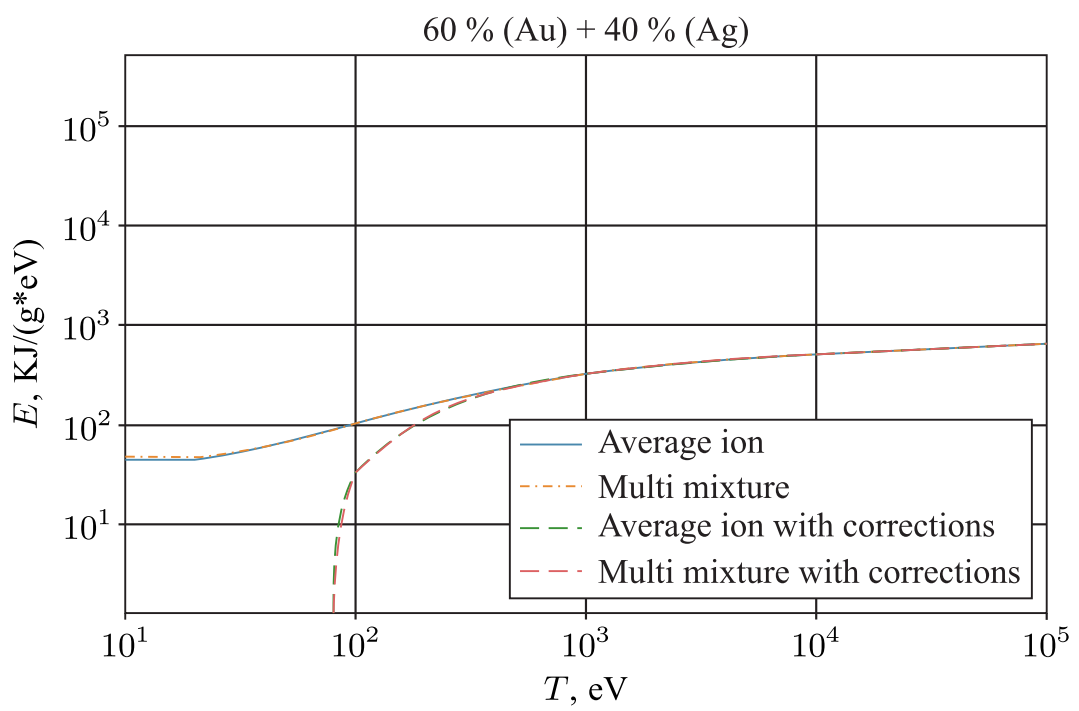

Fig. 6. Entropy of the mixture of gold and silver based on the Thomas-Fermi model with and without quantum-exchange correction at a density of $1 \mathrm{~g} / \mathrm{cm}^{3}$.

Based on the above, it can be concluded that the calculations performed using the two approaches give minor differences, especially in the region where quantum-exchange corrections are neglegible, but the average ion approximation has several advantages. It provides the condition of thermodynamic equilibrium in the field of applicability of quantum exchange-corrections and also has a simpler implementation.

\section{Conclusion}

As a result of the study, two algorithms have been developed and implemented for modelling the thermodynamic parameters of plasma mixture based on the Thomas-Fermi model with quantum-exchange corrections. The approach to solving the system of equations for the ThomasFermi potential with corrections has been proposed for the first time.

As an example of modelling the thermodynamic properties of a substance, gold-silver and beryllium-tungsten mixtures were considered. For them, the distribution of thermodynamic functions and their quantum-exchange corrections were obtained using different approaches. More- 
over, the differences between the approaches in the description of the plasma mixture were discussed. For the first time, the thermodynamic parameters of gold-silver and beryllium-tungsten mixtures were calculated at the selected concentrations.

\section{References}

[1] Zel'dovich, Ya. B. and Raizer, Yu. P., Physics of Shock Waves and High-Temperature Hydrodynamic Phenomena, New York: Dover, 2002, p. 178.

[2] Ryzhkov, S. V. and Chirkov, A. Yu., Alternative Fusion Fuels and Systems, Boca Raton, Fla.: CRC Press, Taylor \& Francis Group, 2019.

[3] Shemyakin, O. P., Levashov, P. R., and Krasnova, P. A., TFmix: A High-Precision Implementation of the Finite-Temperature Thomas - Fermi Model for a Mixture of Atoms, Comput. Phys. Commun., 2019, vol. 235, pp. 378-387.

[4] Kuzenov, V. V. and Shumaev, V. V., Description of the Thermodynamic Properties of Plasma in Saha and Thomas-Fermi Models, Prikl. Fiz., 2015, no. 2, pp. 32-36 (Russian).

[5] Nikiforov, A. F., Novikov, V. G., and Uvarov, V.B., Quantum-Statistical Models of Hot Dense Matter: Methods for Computation Opacity and Equation of State, Progr. Math. Phys., vol. 37, Basel: Birkhäuser, 2005, p. 34.

[6] Kirzhnits, D. A., Quantum Corrections to the Thomas-Fermi Equation, JETP, 1957, vol. 5, no. 1, pp. 64-71; see also: Zh. Ekksper. Teoret. Fiz., 1957, vol. 32, no. 1, pp. 115-123.

[7] Belov, A. A., Golovanov, R. V., Kalitkin, N. N., Kozlitin, I. A., Koryakin, I. A., and Kuzmina, L. V., TEFIS Database. Thermodynamic Functions of Substances, Preprint No. 219, Moscow: KIAM, 2018 (Russian).

[8] Kuzenov, V.V. and Ryzhkov, S. V., Calculation of Plasma Dynamic Parameters of the MagnetoInertial Fusion Target with Combined Exposure, Phys. Plasmas, 2019, vol. 26, no. 9, Art. No. 092704.

[9] Kuzenov, V.V., Ryzhkov, S.V., and Shumaev, V.V., Computer Analysis of Transport, Optical and Thermodynamic Properties of Plasma, High Temp. Mater. Process., 2014, vol. 18, nos. 1-2, pp. 99-109.

[10] Kuzenov, V. V. and Ryzhkov, S. V., Radiation-Hydrodynamic Modeling of the Contact Boundary of the Plasma Target Placed in an External Magnetic Field, Prikl. Fiz., 2014, no. 3, pp. 26-30 (Russian).

[11] Kuzenov, V.V. and Ryzhkov, S. V., Mathematical Modeling of Plasma Dynamics for Processes in Capillary Discharges, Russian J. Nonlinear Dyn., 2019, vol. 15, no. 4, pp. 543-550.

[12] Liberman, M. A. and Johansson, B., Properties of Matter in Ultrahigh Magnetic Fields and the Structure of the Surface of Neutron Stars, Physics-Uspekhi, 1995, vol.38, no.2, pp. 117-136; see also: Uspekhi Fiz. Nauk, 1995, vol. 165, no. 2, pp. 121-142.

[13] Golovanov, R. V. and Lutskii, K. I., Computation of the Integral Fermi-Dirac Function, Math. Models Comput. Simul., 2012, vol. 4, pp. 464-470.

[14] Dyachkov, S. A. and Levashov, P. R., Region of Validity of the Finite-Temperature Thomas - Fermi Model with Respect to Quantum and Exchange Corrections, Phys. Plasmas, 2014, vol.21, no. 5, 052702, 6 pp.

[15] Kuzenov, V. V., Ryzhkov, S. V., and Shumaev, V. V., Numerical Thermodynamic Analysis of Alloys for Plasma Electronics and Advanced Technologies, Probl. Atom. Sci. Tech., 2015, vol. 98, no. 4, pp. 53-56.

[16] Kuzenov, V.V., Ryzhkov, S. V., and Shumaev, V.V., Thermodynamic Properties of Magnetized Plasma Evaluated by Thomas-Fermi Model, Prikl. Fiz., 2014, no. 3, pp. 22-25. 